\title{
TEORES De MetaIS NA BIOMASSA DE Egeria densa, Egeria najas E Ceratophyllum demersum ${ }^{1}$
}

\author{
Metal Contents in Egeria densa, Egeria najas and Ceratophyllum demersum Biomass
}

\author{
CORREAA, M.R. ${ }^{2}$, VELINI, E.D. ${ }^{3}$ e ARRUDA, D.P. ${ }^{4}$
}

\begin{abstract}
RESUMO - Este estudo foi realizado com o objetivo de se estimar o conteúdo de elementos metálicos presentes em três macrófitas aquáticas submersas, procurando avaliar a participação destas plantas na remoção destes elementos na água e criar base de dados que permita prever o impacto ambiental do descarte delas em solo ou na própria água. As amostras foram desidratadas, moídas e encaminhadas aos laboratórios para análise e quantificação dos elementos. Para as três espécies, a maior concentração de elementos foi observada durante o verão, ocorrendo declínio nas concentrações durante o inverno, principalmente para os elementos vanádio e chumbo. Não foi detectada presença do elemento mercúrio em nenhuma das amostras.
\end{abstract}

Palavras-chave: macrófitas aquáticas, controle mecânico, elementos metálicos.

\begin{abstract}
This study was carried out to estimate the metallic element contents in three submerged aquatic macrophytes, seeking to evaluate the participation of these plants in metal removal in the water and to create a data base to predict the environmental impact of the discard in soil or in the water, itself. Samples were dried, milled and taken to laboratories for element analysis and quantification. The highest concentrations of elements for the three species were observed during the summer, with a decrease in the winter, mainly for Vanadium and lead. Mercury was not detected in the samples.
\end{abstract}

Key words: aquatic macrophytes, mechanical control, metallic elements.

\section{INTRODUÇÃO}

A intensificação de atividades antrópicas nas bacias hidrográficas, normalmente sem planejamento ou controle, associada ao carregamento de nutrientes para o leito dos rios, através do aporte de despejos domésticos, industriais e fertilizantes químicos empregados nos cultivos ao longo de toda a bacia hidrográfica, tem levado a uma condição de desequilíbrio no sistema, caracterizado pela grande disponibilidade de nutrientes, acelerando o crescimento de organismos autotróficos, particularmente algas plantônicas (fitoplâncton) e macrófitas aquáticas (Valente et al., 1997; Velini, 2000).

Tanaka (1998) e Velini (2000) reportam que o processo de eutrofização dos sistemas aquáticos pode acarretar vários prejuízos à pesca e recreação, além do entupimento das tomadas de água de turbinas de usinas hidrelétricas.

A alta incidência de vegetação aquática nos reservatórios das usinas tem levado ao estudo de alternativas para o manejo e controle dessas plantas. O controle mecânico visa a retirada do material vegetal do meio hídrico para

Recebido para publicação em 23/11/2001 e na forma revisada em 4/1/2002.

2 Eng.-Agr., aluno Pós-graduação, Dep. Produção Vegetal/Agricultura, FCA-UNESP, Caixa Postal 237, 18603-970 BotucatuSP, <mrcorrea@fca.unesp.br>; ${ }^{3}$ Prof. Dr., Dep. Produção Vegetal/Agricultura, FCA-UNESP, Caixa Postal 237, 18603-970 Botucatu-SP. ${ }^{4}$ Estudante do curso de Química, Universidade do Sagrado Coração, Bauru-SP. 
posterior descarte em ambiente terrestre. No entanto, pouco se sabe sobre as características químicas do material, bem como sobre a segurança ambiental na área de descarte, em relação à liberação de elementos tóxicos ao solo e lençol freático, no momento e após o descarte da biomassa.

Em sistemas aquáticos, destaca-se um grupo de elementos químicos, denominados metais, que compreendem 84 elementos com densidade superior a $5 \mathrm{~g} \mathrm{~cm}^{-3}$, bons condutores de calor e eletricidade (Moraes, 1999). Embora a palavra metal pesado esteja associada com contaminação, em estudos ambientais a conotação de toxicidade geralmente relaciona-se com a concentração das substâncias. Essa estimativa, entretanto, pode não fornecer a disponibilidade e o nível de toxicidade para organismos aquáticos (Moraes, 1999; Lewander, 1996) ou estabelecer limites para a concentração de metais no sedimento (Kähkömen et al., 1997).

A contaminação do ambiente por metais potencialmente tóxicos tem se tornado preocupante devido a utilização de grande variedade desses elementos pela indústria. As macrófitas aquáticas possuem absorção seletiva de certos íons e têm sido largamente utilizadas como bioindicadores, pela habilidade em integrar e monitorar variações nas concentrações e elementos no meio hídrico.

O conhecimento das interações entre elementos químicos, água, sedimentos e plantas aquáticas é de certa forma incompleto. A falta de informações refere-se à complexidade em estudar todos os fatores ambientais envolvidos que afetam a disponibilidade dos elementos para as plantas aquáticas.

Dessa forma, este estudo objetivou quantificar os principais elementos metálicos presentes em três macrófitas aquáticas submersas, sendo as coletas realizadas no reservatório da usina hidrelétrica de Jupiá.

\section{MATERIAL E MÉTODOS}

Amostras de E. densa, E. najas e C. demersum foram coletadas em duas épocas do ano, verão e inverno, em vários pontos da porção Tietê do reservatório da usina hidrelétrica de Jupiá, envolvendo quatro lagoas marginais e o leito do rio em questão, correspondente ao canal de eclusagem da Usina de
Três Irmãos até a confluência com o rio Paraná, no município de Itapura. As plantas foram coletadas com auxílio de um gancho, em bancos homogêneos de colonização, sendo colocadas em sacos plásticos com água, mantendo-se a umidade destas durante o transporte.

Em laboratório, as amostras foram separadas por espécie e lavadas em água para remoção do material contaminante aderido durante as coletas. Em seguida, após breve secagem para retirada de excesso de água, as plantas foram acondicionadas em sacos de papel e colocadas em estufa de circulação forçada de ar a uma temperatura de $60^{\circ} \mathrm{C}$ por um período de sete dias, sendo posteriormente moídas em micromoinho, etiquetadas e encaminhadas para análise dos elementos metálicos.

A determinação dos elementos químicos foi realizada no laboratório de relação solo-planta do Departamento de Produção Vegetal, Setor Agricultura, da FCA-Unesp/Botucatu. Para determinação dos teores dos elementos, o material foi digerido com uma mistura de ácido nítrico e ácido perclórico, seguindo metodologia proposta por Malavolta et al. (1989). Foram digeridas amostras em branco, para posterior correção dos valores.

As amostras digeridas foram submetidas a espectrometria de plasma para determinação direta das concentrações de $\mathrm{Na}, \mathrm{Mn}, \mathrm{Ba}, \mathrm{Ni}, \mathrm{V}$, $\mathrm{Se}, \mathrm{Cr}, \mathrm{As}, \mathrm{Pb}, \mathrm{Li}, \mathrm{Sn}, \mathrm{Cd}$ e Hg, de acordo com soluções-padrão preestabelecidas no laboratório. Os valores fornecidos pelo aparelho foram corrigidos de acordo com as concentrações utilizadas na digestão e a solução em branco, obtendo-se, assim, os teores finais de cada elemento presente nas amostras.

Foram determinadas as diferenças nas concentrações dos elementos metálicos nas macrófitas aquáticas, considerando-se as épocas de amostragem e a espécie de plantas. Os resultados foram submetidos à análise de variância, comparando-se as médias dos tratamentos com auxílio do teste "t" a 10\% de probabilidade.

\section{RESULTADOS E DISCUSSÃO}

Maiores concentrações de sódio foram observadas na espécie E. najas em ambas as amostragens (verão e inverno), sendo 
estatisticamente superior às das demais espécies de plantas (Tabelas 1 e 2). Como observado na Tabela 3, os teores de sódio foram significativamente mais elevados nas plantas coletadas no verão do que nas coletadas no inverno. Wetzel (1993) reporta que a distribuição espacial e temporal deste elemento é uniforme, apresentando variações sazonais muito pequenas, e a redução em suas concentrações está presumidamente relacionada com a utilização deste elemento pelas algas e macrófitas aquáticas.

Teores de manganês foram superiores em C. demersum, tanto no verão (Tabela 1), sendo significativamente superiores aos das demais espécies, quanto no inverno (Tabela 2). De acordo com os dados da Tabela 3 , nota-se aumento significativo dos teores médios deste elemento nas plantas coletadas no inverno, sendo esse aumento significativo apenas para E. najas. Para C. demersum, os teores de manganês foram mais elevados nas plantas coletadas no verão do que no inverno.

As espécies de Egeria mostraram concentrações de bário significativamente superiores à encontrada em C. demersum, tanto nas coletadas no verão (Tabela 1) quanto no inverno (Tabela 2). Entretanto, apesar de haver variações nos teores, não foram observadas diferenças estatísticas nos teores deste elemento nas plantas amostradas no verão em relação ao inverno (Tabela 3).

As diferenças de concentrações de níquel (nas três espécies de plantas estudadas) não foram significativas nas amostragens realizadas no período de verão, como pode ser visto na Tabela 1. Por outro lado, nas coletas realizadas no inverno, C. demersum apresentou teor médio $44 \%$ superior ao obtido em $E$. densa (Tabela 2). Observando os dados da Tabela 3, nota-se que há decréscimo nos teores deste elemento nas plantas coletadas no verão em relação às coletadas no inverno, o que também ocorreu com os teores de vanádio. Entretanto, apenas C. demersum não apresentou diferença significativa nos teores de níquel, quando realizada a comparação entre as épocas de amostragem. Nos teores de arsênio, também foi observada redução nas amostragens realizadas no verão, porém não tão expressiva quanto Ni e V (Tabela 3).

Tabela 1 - Teores médios de elementos metálicos em plantas aquáticas imersas presentes no reservatório de Jupiá. Coleta realizada em 8 de janeiro de 2001, em nove locais ao longo do rio Tietê. Botucatu-SP, 2001

\begin{tabular}{|c|c|c|c|c|c|c|c|c|c|c|c|c|}
\hline \multirow{2}{*}{ Espécie/Elemento } & \multicolumn{12}{|c|}{$\mathrm{mg} \mathrm{kg}^{-1}$ de Matéria Seca } \\
\hline & $\mathrm{Na}$ & $\mathrm{Mn}$ & $\mathrm{Ba}$ & $\mathrm{Ni}$ & $\mathrm{V}$ & $\mathrm{Se}$ & $\mathrm{Cr}$ & As & $\mathrm{Pb}$ & $\mathrm{Li}$ & Sn & $\mathrm{Cd}$ \\
\hline Egeria densa & $4723,7 \mathrm{~b}$ & $3671,4 \mathrm{~b}$ & 396,1 a & $25,7 \mathrm{a}$ & $20,8 \mathrm{a}$ & $4,6 \mathrm{ab}$ & $3,9 \mathrm{ab}$ & $3,5 \mathrm{a}$ & $2,5 \mathrm{a}$ & $1,1 \mathrm{a}$ & $1,0 \mathrm{a}$ & 0,18 a \\
\hline Egeria najas & $6733,5 \mathrm{a}$ & $2795,1 \mathrm{~b}$ & 439,8 a & $23,2 \mathrm{a}$ & 19,9 a & $8,3 \mathrm{a}$ & $3,0 \quad b$ & $2,8 \mathrm{a}$ & $3,1 \mathrm{a}$ & $1,1 \mathrm{a}$ & $0,9 \mathrm{a}$ & $0,17 \mathrm{ab}$ \\
\hline C. demersum & $2382,8 \mathrm{c}$ & $6444,9 \mathrm{a}$ & $257,3 \mathrm{~b}$ & $27,9 \mathrm{a}$ & $19,5 \mathrm{a}$ & $1,2 \mathrm{~b}$ & $4,3 \mathrm{a}$ & $3,6 \mathrm{a}$ & $3,9 \mathrm{a}$ & $0,9 \mathrm{a}$ & $1,0 \mathrm{a}$ & $0,09 \mathrm{~b}$ \\
\hline CV $(\%)$ & 21,344 & 51,299 & 24,060 & 31,201 & 22,746 & 37,924 & 33,372 & 73,044 & 56,617 & 83,712 & 91,556 & 59,186 \\
\hline F & $42,03^{*}$ & $5,83^{*}$ & $10,16^{*}$ & $0,68^{\mathrm{ns}}$ & $0,17^{\mathrm{ns}}$ & $7,57^{\mathrm{ns}}$ & $1.93^{\text {ns }}$ & $0,22^{\text {ns }}$ & $1,43^{\text {ns }}$ & $0,08^{\text {ns }}$ & $0,01^{\mathrm{ns}}$ & $1,99^{\mathrm{ns}}$ \\
\hline
\end{tabular}

Médias seguidas de mesma letra nas colunas não diferem estatisticamente a $10 \%$ de probabilidade.

* Significativo a $5 \%$ de probabilidade $\mathrm{e}^{\text {ns }}$ não-significativo.

Tabela 2 - Teores médios de elementos metálicos em plantas aquáticas imersas presentes no reservatório de Jupiá. Coleta realizada em 24 de julho de 2001, em nove locais ao longo do rio Tietê. Botucatu-SP, 2001

\begin{tabular}{|l|c|c|c|c|c|c|c|c|c|c|c|c|}
\hline \multirow{2}{*}{ Espécie/Elemento } & \multicolumn{10}{|c|}{$\mathrm{mg} \mathrm{kg}^{-1}$ de Matéria Seca } \\
\cline { 2 - 12 } & $\mathrm{Na}$ & $\mathrm{Mn}$ & $\mathrm{Ba}$ & $\mathrm{Ni}$ & $\mathrm{V}$ & $\mathrm{Se}$ & $\mathrm{Cr}$ & $\mathrm{As}$ & $\mathrm{Pb}$ & $\mathrm{Li}$ & $\mathrm{Sn}$ & $\mathrm{Cd}$ \\
\hline Egeria densa & $2292,7 \mathrm{~b}$ & $4839,0 \mathrm{a}$ & $359,8 \mathrm{a}$ & $6,5 \mathrm{~b}$ & $2,7 \mathrm{a}$ & $2,9 \mathrm{a}$ & $2,6 \mathrm{a}$ & $1,3 \mathrm{a}$ & $0,6 \mathrm{a}$ & $0,7 \mathrm{a}$ & $0,9 \mathrm{a}$ & $0,03 \mathrm{a}$ \\
Egeria najas & $3336,9 \mathrm{a}$ & $4150,2 \mathrm{a}$ & $371,0 \mathrm{a}$ & $8,5 \mathrm{ab}$ & $3,2 \mathrm{a}$ & $1,8 \mathrm{a}$ & $2,5 \mathrm{a}$ & $1,5 \mathrm{a}$ & $0,4 \mathrm{a}$ & $0,8 \mathrm{a}$ & $0,6 \mathrm{a}$ & $0,04 \mathrm{a}$ \\
C. demersum & $1581,7 \mathrm{c}$ & $5968,3 \mathrm{a}$ & $254,3 \mathrm{~b}$ & $11,6 \mathrm{a}$ & $6,1 \mathrm{a}$ & $1,6 \mathrm{a}$ & $3,8 \mathrm{a}$ & $0,7 \mathrm{a}$ & $0,0 \mathrm{~b}$ & $1,3 \mathrm{a}$ & $1,2 \mathrm{a}$ & $0,15 \mathrm{a}$ \\
\hline $\mathrm{CV}(\%)$ & 28,389 & 44,102 & 36,745 & 67,507 & 90,4228 & 35,212 & 61,731 & 74,076 & 37,983 & 93,145 & 78,798 & 211,933 \\
$\mathrm{~F}$ & $14,52^{*}$ & $1,50^{\mathrm{ns}}$ & $2,52^{*}$ & $1,62^{\mathrm{ns}}$ & $0,76^{\mathrm{ns}}$ & $1,72^{\mathrm{ns}}$ & $1,28^{\mathrm{ns}}$ & $2,85^{*}$ & $0,46^{\mathrm{ns}}$ & $0,95^{\mathrm{ns}}$ & $0,75^{\mathrm{ns}}$ & $0,15^{\mathrm{ns}}$ \\
\hline
\end{tabular}

Médias seguidas de mesma letra nas colunas não diferem estatisticamente a $10 \%$ de probabilidade.

* Significativo a $5 \%$ de probabilidade e ${ }^{\text {ns }}$ não-significativo. 
Tabela 3 - Teores médios de elementos metálicos em plantas aquáticas imersas presentes no reservatório de Jupiá. Coletas realizadas em 8 de janeiro (verão) e 24 de julho (inverno) de 2001, em nove locais ao longo do rio Tietê. Botucatu-SP, 2001

\begin{tabular}{|c|c|c|c|c|c|c|c|c|c|c|c|c|}
\hline \multirow{2}{*}{ Espécie/Elemento } & \multicolumn{12}{|c|}{$\mathrm{mg} \mathrm{kg}^{-1}$ de Matéria Seca } \\
\hline & $\mathrm{Na}$ & $\mathrm{Mn}$ & $\mathrm{Ba}$ & $\mathrm{Ni}$ & $\mathrm{V}$ & $\mathrm{Se}$ & $\mathrm{Cr}$ & As & $\mathrm{Pb}$ & $\mathrm{Li}$ & $\mathrm{Sn}$ & $\mathrm{Cd}$ \\
\hline 6. & $23,7 \mathrm{a}$ & $3671,4 \mathrm{a}$ & $396,1 \mathrm{a}$ & $25,7 \mathrm{a}$ & $20,8 \mathrm{a}$ & $4,6 \mathrm{a}$ & $3,9 \mathrm{a}$ & $3,5 \mathrm{a}$ & $2,5 \mathrm{a}$ & $1,1 \mathrm{a}$ & $1,0 \mathrm{a}$ & $0,18 \mathrm{a}$ \\
\hline E. de & $92,7 \mathrm{~b}$ & $4839,0 \mathrm{a}$ & $359,8 \mathrm{a}$ & $6,5 \mathrm{~b}$ & $2,7 \mathrm{~b}$ & $2,9 \mathrm{a}$ & $2,6 \mathrm{~b}$ & $1,3 \mathrm{a}$ & $0,6 \mathrm{a}$ & $0,7 \mathrm{a}$ & $0,9 \mathrm{a}$ & $0,03 \mathrm{a}$ \\
\hline $\mathrm{CV}(\%)$ & 23,269 & 44,928 & 28,394 & 35,542 & 17,013 & 39,687 & 33,627 & 110,785 & 88,804 & 101,054 & 17,145 & 82,444 \\
\hline $\mathrm{F}$ & $39,91^{*}$ & $1,68^{\mathrm{ns}}$ & $0,51^{\mathrm{ns}}$ & $50,33^{*}$ & $96,48^{*}$ & $1,41^{\mathrm{ns}}$ & $6,39^{*}$ & $1,77^{\mathrm{ns}}$ & $1,58^{\mathrm{ns}}$ & $0,63^{\mathrm{ns}}$ & $0,57^{\mathrm{ns}}$ & $1,07^{\mathrm{ns}}$ \\
\hline E. najas $(\mathrm{V}$ & $6733,5 \mathrm{a}$ & $2795,1 \mathrm{~b}$ & $439,8 \mathrm{a}$ & $23,2 \mathrm{a}$ & $19,9 \mathrm{a}$ & $8,3 \mathrm{a}$ & $3,0 \mathrm{a}$ & $2,8 \mathrm{a}$ & $3,1 \mathrm{a}$ & $\overline{1,1 \mathrm{a}}$ & $0,9 \mathrm{a}$ & $0,17 \mathrm{a}$ \\
\hline E. $n a$ & $3336,9 \mathrm{~b}$ & $4150,2 \mathrm{a}$ & $371,0 \mathrm{a}$ & $8,5 \mathrm{~b}$ & $3,2 \mathrm{~b}$ & $1,8 \mathrm{a}$ & $2,5 \mathrm{a}$ & $1,5 \mathrm{a}$ & $0,4 \mathrm{a}$ & $0,8 \mathrm{a}$ & $0,6 \mathrm{a}$ & $0,04 \mathrm{a}$ \\
\hline $\mathrm{CV}(\%)$ & 19,271 & 37,482 & 27,635 & 38,164 & 20,438 & 27,579 & 49,462 & 68,730 & 75,235 & 77,462 & 118,383 & 49,614 \\
\hline F & $47,87^{*}$ & $3,94^{*}$ & $1,42^{\mathrm{ns}}$ & $23,64^{*}$ & $62,95^{*}$ & $23,83^{\mathrm{ns}}$ & $0,50^{\mathrm{ns}}$ & $2,45^{\mathrm{ns}}$ & $1,43^{\mathrm{ns}}$ & $0,80^{\mathrm{ns}}$ & $0,23^{\mathrm{ns}}$ & $2,39^{\text {ns }}$ \\
\hline C. den & $2382,8 \mathrm{a}$ & 6444,9 a & $257,3 \mathrm{a}$ & $27,9 \mathrm{a}$ & $19,5 \mathrm{a}$ & $1,2 \mathrm{a}$ & $4,3 \mathrm{a}$ & $3,6 \mathrm{a}$ & $3,9 a$ & $0,9 \mathrm{a}$ & $1,0 \mathrm{a}$ & $0,09 \mathrm{a}$ \\
\hline C. de & $1581,7 \mathrm{~b}$ & $5968,3 \mathrm{a}$ & $254,3 \mathrm{a}$ & $11,6 \mathrm{a}$ & $6,1 \mathrm{~b}$ & $1,6 \mathrm{a}$ & $3,8 \mathrm{a}$ & $0,7 \mathrm{~b}$ & $0,0 \mathrm{~b}$ & $1,3 \mathrm{a}$ & $1,2 \mathrm{a}$ & $0,15 \mathrm{a}$ \\
\hline $\mathrm{CV}(\%)$ & 35,284 & 48,616 & 37,784 & 45,289 & 38,492 & 39,554 & 50,623 & 56,634 & 31,677 & 83,234 & 71,814 & 82,311 \\
\hline 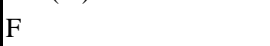 & $5,90^{*}$ & $0,11^{\mathrm{ns}}$ & $0,002^{*}$ & $15,04^{*}$ & $10,53^{*}$ & $0,44^{\mathrm{ns}}$ & $0,21^{\mathrm{ns}}$ & $11,12^{*}$ & 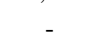 & $0,54^{\mathrm{ns}}$ & $0,05^{\mathrm{ns}}$ & $0,73^{\mathrm{ns}}$ \\
\hline
\end{tabular}

Médias seguidas de mesma letra nas colunas não diferem estatisticamente a $10 \%$ de probabilidade.

* Significativo a $5 \%$ de probabilidade e ${ }^{\text {ns }}$ não-significativo.

Kähkömen et al. (1997) puderam observar teores de Ni semelhantes aos encontrados nas plantas coletadas no verão, em folhas de E. canadensis. Por outro lado, Ledl et al. (1981), avaliando esta mesma espécie, em um rio da Áustria, observaram teores de 3 a $5 \mu \mathrm{g} \mathrm{g}^{-1} \mathrm{MS}$, semelhantes aos observados nas plantas de Egeria coletadas no inverno (Tabela 2).

A maior concentração de selênio foi detectada em E. najas coletada no verão (Tabela 1). No entanto, apesar de haver decréscimo nas concentrações médias deste elemento em E. najas e E. densa e acréscimo em C. demersum, não foram observadas diferenças estatísticas significativas entre as épocas de amostragem.

Plantas de C. demersum coletadas no verão apresentaram maior conteúdo de cromo, sendo estatisticamente superior ao de E. najas, com menor teor médio observado. Houve decréscimo nos teores médios nas plantas coletadas no inverno em relação ao verão, porém somente em E. densa foi significativo (Tabela 3).

Analisando os teores de chumbo em plantas coletadas no verão (Tabela 1), a maior concentração foi observada em C. demersum. Entretanto, no inverno (Tabela 2), este elemento não foi detectado nas estruturas desta espécie, havendo redução nos teores médios das demais espécies avaliadas. Lewander et al. (1996), estudando macrófitas aquáticas como indicadores de metais em partes poluídas do rio Przesza, verificaram concentrações médias de chumbo, em Myriophyllum verticillatum, em torno de $26 \mu \mathrm{g} \mathrm{g}^{-1} \mathrm{MS}$, valores estes consideravelmente superiores aos observados no presente trabalho.

Os elementos lítio e estanho apresentaram teores semelhantes nas espécies de plantas avaliadas, apresentando decréscimo não-significativo nas concentrações, quando comparadas com plantas coletadas no inverno e verão. Somente C. demersum mostrou pequeno acúmulo no teor de estanho nas plantas coletadas no inverno. Esta espécie apresentou, também, acréscimo na concentração de cádmio, enquanto $E$. densa e E. najas tiveram acentuado decréscimo, embora não-significativo, nas plantas amostradas no inverno (Tabela 3). Esses valores estão abaixo dos obtidos por Lewander et al. (1996) em M. verticillatum, com média de $32 \mu \mathrm{g} \mathrm{g}^{-1} \mathrm{MS}$.

Não foi detectada, em nenhuma das amostras de plantas coletadas no inverno ou verão, a presença do elemento mercúrio.

\section{LITERATURA CITADA}

KÄHKÖMEN, M. A.; PANTSAR-KALLI, M.; MANNINEN, P. K. G. Analysing heavy metal concentration in the different parts of Elodea canadensis and surface sediment with PCA in two boreal lakes in southern Finland. Chemosphere, v. 35, n. 11, p.2645-2656, 1997. 
LEDL, G.; JANAUER, G. A.; HORAK, O. Die Anricherung von Schwermetallen in Wasserpfanzen aus einiger österreichichen. Acta Hydrochim. Hidrobiol., n. 9, v. 6, p.651-663, 1981.

LEWANDER, M. et al. Macrophytes as indicator of bioavailable $\mathrm{Cd}, \mathrm{Pb}$ and $\mathrm{Zn}$ flow in the river Przemsza, Katowice Region. Appl. Geochem., v. 11, p.169-173, 1996.

MALAVOLTA, E.; VITTI, G. C.; OLIVEIRA, S. A. Avaliação do estado nutricional das plantas - Princípios e aplicações. Piracicaba: Fundação Brasileira para Pesquisa da Potassa e do Fosfato, 1989. 201 p.

MORAES, A. R. Estimativa de estoque de elementos químicos em macrófitas aquáticas do reservatório de Salto Grande (Americana-SP). São Carlos: EESC, 1999. 94 p. Dissertação (Mestrado em Ciências da Engenharia Ambiental) - Universidade de São Paulo, 1999.
TANAKA, R. H. Prejuízos provocados pelas plantas aquáticas. In: WORSHOP CONTROLE DE PLANTAS AQUÁTICAS, 1998, Brasília. Resumos... Brasília: IBAMA, 1998. p. 36-38.

VALENTE, J. P. S.; PADILHA, P. M.; SILVA, A. M. M. Contribuição da cidade de Botucatu-SP com nutrientes (fósforo e nitrogênio) na eutrofização da represa de Barra Bonita. Eclét. Quím., v. 22, p. 31-48, 1997.

VELINI, E. D. Controle de plantas daninhas aquáticas. In: CONGRESSO BRASILEIRO DA CIÊNCIA DAS

PLANTAS DANINHAS, 22., 2000, Foz do Iguaçu. Palestras... Foz do Iguaçu: Sociedade Brasileira da Ciência das Plantas Daninhas, 2000. p. 137-147.

WETZEL, R. G. Limnologia. 2.ed. Lisboa: Saunders College Publishing, 1993. 919 p. 